\title{
Re: Outcomes of Endoscopic Realignment of Pelvic Fracture Associated Urethral Injuries at a Level 1 Trauma Center
}

\author{
Laura S. Leddy, Alex J. Vanni, Hunter Wessells, Bryan B. Voelzke
}

University of Washington Medical Center, Harborview Medical Center, Department of Urology, Washington, USA

J Urol 2012;188:174-178. doi: 10.1016/j.juro.2012.02.2567. Epub 2012 May 15.

\section{EDITORIAL COMMENT}

This paper is about the success of early endoscopic (retrorgrade or retrograde-antegrade combined) realignment of pelvic fracture associated urethral injury after blunt pelvic trauma As known, immediate management of pelvic fracture associated urethral injury is to place a suprapubic catheter to provide urinary drainage. Thereafter, 2 alternative management approaches are available. The first one is to perform an internal urethroplasty some months later. The alternative is early endoscopic urethral realignment (1).

The authors rewieved endoscopic realignment procedures in 19 patients. Twelve of them had complete and 4 patients had incomplete urethral disruption. However, preoperative status of the remaining 3 patients could not be determined before surgery. The mean time to realignment and the mean duration of urethral catheterization after realignment were 2 and 53 days, respectively. The follow-up protocol included uroflowmetry, post-void residual urine measurement and cystoscopic evaluation. Failure of the procedure was defined as requirement of urethral dilatation, direct vision internal urethrotomy, posterior urethroplasty or self-catheterization after initial urethral catheter removal.

The authors reported that early endoscopic realignment was unsuccesful in 15 of 19 patients (78.9\%). These patients required intervention with a mean of 79 days after catheter removal. They were managed by posterior urethroplasty or internal urethrotomy.

The authors concluded that early endoscopic realignment causes sympathetic urethral strictures. Close follow-up is necessary after catheter removal because it usually requires additional intervention.

\section{REFERENCE}

1. Gomez RG, Mundy T, Dubey D, El-Kassaby AW, Firdaoessaleh, Kodama R, Santucci R. SIU/ICUD Consultation on Urethral Strictures: Pelvic fracture urethral injuries. Urology 2014;83(3 Suppl):48-58. 